\title{
A STUDY ON EFFECT OF FUNCTIONAL GRADATION ON ROTATING DISC
}

\author{
Divin Kotnise ${ }^{1}$, Naveen Bharadishettar ${ }^{2}$ \\ ${ }^{I}$ Student, Department of Mechanical Engineering, MVJCE, Bangalore, Karnataka, India \\ ${ }^{2}$ Assistant Professor, Department of Mechanical Engineering, MVJCE, Bangalore, Karnataka, India
}

\begin{abstract}
Finite Element Method (FEM) is a new field of analysis which has become a steadfast method, as applied to Thermal and structural analysis. By the application of Finite Element Analysis in day to day design tasks reduces cost effectively, with capabilities spreading a broad scope in industries. As academics and increase in commercial software becoming accessible, it turns into basic tool for effective analysis. FEM enables the evolution of quality products within a short design cycle with less number of field failure depending on the skill of the analyst. In this paper study of radial stress distribution between two different materials i.e., a homogeneous rotating disc compared with material of Functional grading are done and respective results are compared. The first case of gradation is by varying the poison's ratio linearly and other is by parabolic variation. Resulting radial stress equation is derived by applying the linear variation and the related boundary conditions. Results are amply illustrated by defining coding by applying boundary conditions and then contours plotted with respective results.
\end{abstract}

Keywords - Radial Stress, Poisson's Ratio, Finite Element Method (FEM).

\section{BACKGROUND}

In Japan in middle of 1980, the concept of Functionally Graded Materials (FGM) was conceptualized. This FGM found its multipurpose utility in various engineering manufacturing units. The concept of FGM was well utilised in the manufacture of the rocket science for the material stability to withstand a 1500-degree temperature. Thus the FGM plays a vital role from manufacture of non-corrosive material pin to the aero-space engineering. The necessity to produce an effective material for flywheel, development was made possible by use of FGM by compositional and/or micro structural gradients within the same material which had the prominent property of grading which lead towards one, two or three dimensions of the material. The FGM found to be more competent when compared to the classical composites. Hence an attempt to comment on performance of the flywheel with FGM is made. The FGM enhanced materials resulted in effective performance in metal industries commencing from the manufacture of Main Battle Tank to Space science. The technological application of FGM became industry oriented especially infused in manufacture of huge aircraft, space industry, thermal, coating for turbines, engines, super-hard cutting tools, artificial bones etc. thus superseding the classical composites used in industries.

\section{STRESS ANALYSIS}

The quality of the product depends primarily on the safe and reliable design. Before attempting to proceed with production, it is necessary to monitor the stresses induced in the component during working condition and comprehensive study of stress distribution in the specimen. Generally, when a circular disc rotates the centrifugal force act on the disc following which tensile and bending stress are induced on a rim of flywheel ${ }^{[6]}$. Thus, optimum working ofis attributed to mainly three factors, i.e., material strength, geometry (crosssection) and rotational speed.

Analytical solution involves mathematical modelling of stress equations for functionally Gradient Material by varying the Poisson's ratio as a functionally grading parameter and simulation is used for comparing results, a rotating disc of homogeneous composition and Functionally Graded composition is modelled as a solid disc with central hole in Catia and analysed using analytical method with a suitable programme code and results are verified using Computer Aided Engineering (CAE) software such as Altair's Hypermesh version 13 for Pre-processing and analysis results are obtained using Altair's Optistruct.

\section{MODELLING}

For initial investigation the flywheel is approximated and modelled as a circular hollow disc so that it is capable of mounting on a rotating shaft. In this operation, modelling is executed by using CATIA. The disc is modelled with dimensions such as

- $\quad$ Disc diameter $=100 \mathrm{~mm}$

- Hole diameter for shaft assembly $=20 \mathrm{~mm}$

- $\quad$ Thickness $=5 \mathrm{~mm}$. 


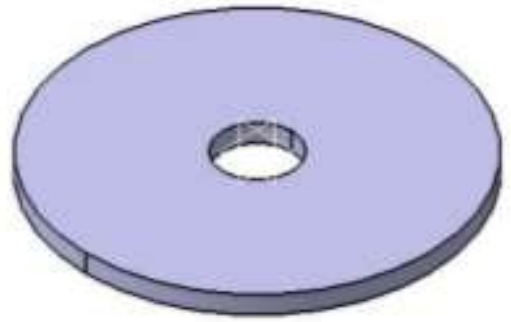

Fig-1: Geometry of disc

\section{ANALYTICAL SOLUTION}

Analytical solutions for homogeneous material are through equations of stress and strain derived by the first principles of elasticity usually of the closed form. In rotating disc, the stresses induced are in radial and tangential direction.

Rotating disc are cylindrical members mounted on shafts connected to prime mover. Practically stress distribution is of great importance as in adverse cases of high speeds, it may lead to catastrophic failure of mechanical system. Stress distribution in rotating disc is thickness dependent that is if the thickness is lesser than the radius of cylindrical member, radial and tangential stresses can be neglected and the definition of problem yields a simple solution.

Radial stress $\left(\sigma_{\mathrm{r}}\right)$ and tangential stress equations $\left(\sigma_{\theta}\right)$ for a rotating disc is given by

$\sigma_{\mathrm{r}}=\frac{(3+v)}{8} \rho \omega^{2}\left(\mathrm{~b}^{2}+\mathrm{a}^{2}-\frac{a^{2} b^{2}}{r^{2}} \mathrm{r}^{2}\right)$

$\sigma_{\theta}=\frac{(3+v)}{8} \rho \omega^{2}\left(\mathrm{~b}^{2}+\mathrm{a}^{2}+\frac{a^{2} b^{2}}{r^{2}}-\frac{(1+3 v)}{(3+v)} \mathrm{r}^{2}\right)$

To functionally grade the material by choosing poison's ratio as a functional grading parameter, the linear relation of variation is given by

$$
v=v_{0}+v_{1} r(a)
$$

For Simplification, Let

$$
v=\mathrm{k}
$$

Therefore, equation (a) becomes

$$
\mathrm{k}=\mathrm{k}_{0}+\mathrm{k}_{1} \mathrm{r}(\mathrm{b})
$$

The Relationship between the Radial and tangential stress of a homogeneous disc is given by

$$
\left[\sigma_{\theta}-\sigma_{\mathrm{r}}\right](1+v)+\mathrm{r} \frac{d}{d r}\left(\sigma_{\theta}\right)-\mathrm{r} \frac{d}{d r}\left(v \sigma_{r}\right)=0
$$

On substituting equation (b) in equation (2) and respective simplification results in equation

$$
\mathrm{r}^{2} \frac{d^{2} \sigma_{r}}{d r^{2}}+3 \mathrm{r} \frac{d \sigma_{r}}{d r}-\mathrm{rd} \sigma_{\mathrm{r}}+\left[3+\mathrm{k}_{0}+\mathrm{k}_{1} \mathrm{r}\right] \rho \omega^{2} \mathrm{r}^{2}=0
$$

Equation (3) is of the Bessel equation form and represents the radial $\left(\sigma_{\mathrm{r}}\right)$ stress equation for a functional graded rotating disc with linear variation of poison's ratio $(v)$.

\section{FINITE ELEMENT ANALYSIS}

The process of generating Finite Element model consists of meshing or discretizing the model into corresponding elements and the analysis setup with respect to the solver by which it is desired to obtain the analysis results. In brief the process is discussed below. The analysis is branched into two cases i.e., homogeneous disc and functionally graded disc.

\subsection{Pre - Processing}

It involves discretization of Model into nodes and elements of required finite size. The process of discretization is known as meshing. Mesh of a model depends on various factors like thickness, element type and type of results and degree of accuracy. Based on these factors an effective mesh is generated to yield desired results.

In disc model, a solid mesh of hex-mesh elements is generated with an average element size of $2 \mathrm{~mm}$ for each tetra element. Thus the CAD model is discretized into a total of 27600 nodes, creating 22000 elements. It is desired to have at least two layers of elements if the thickness is significant. Mesh density study is carried out such that optimum size is selected if the variation of results obtained by two consecutive mesh densities have a variation of $5 \%$. Thus results obtained by the optimum mesh density is considered to be accurate. The discretized finite element model is shown in the figure 2(a) below.

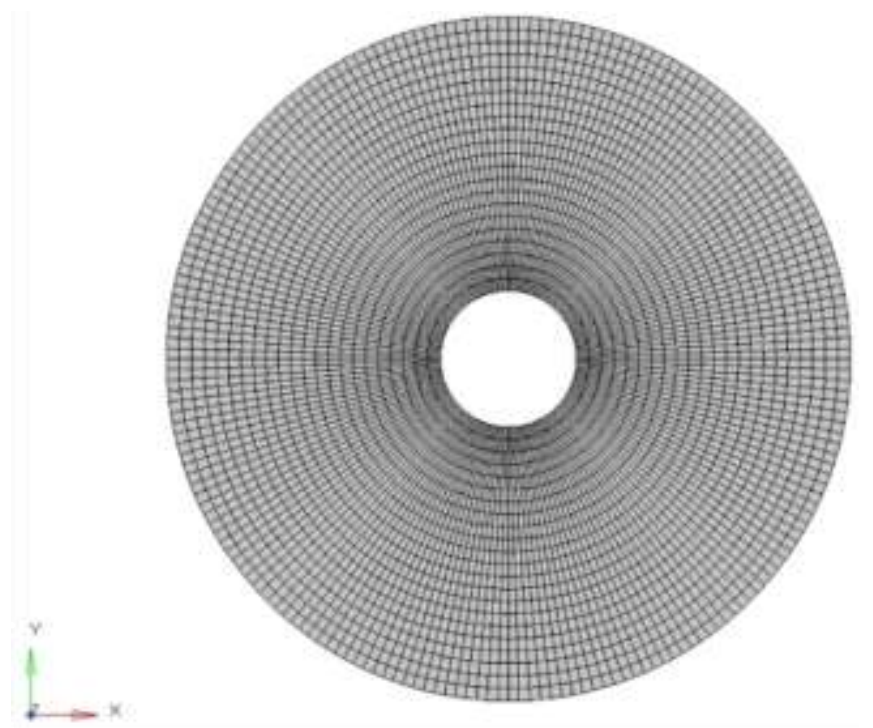

Fig - 2(a): Meshed model - Homogeneous disc

For the analysis of Functional Gradient Material, the specimen in the CAD model is divided into eleven equal parts along the radius. Appropriate hex-element mesh is generated with the Load and System collectors remaining the same. 
Functional gradation is by varying the poisons ratio in each discretized geometrical member across the radius as shown in figure 2(b), thereby the entire model is transformed into a heterogeneous material model with the material varying across the radius.

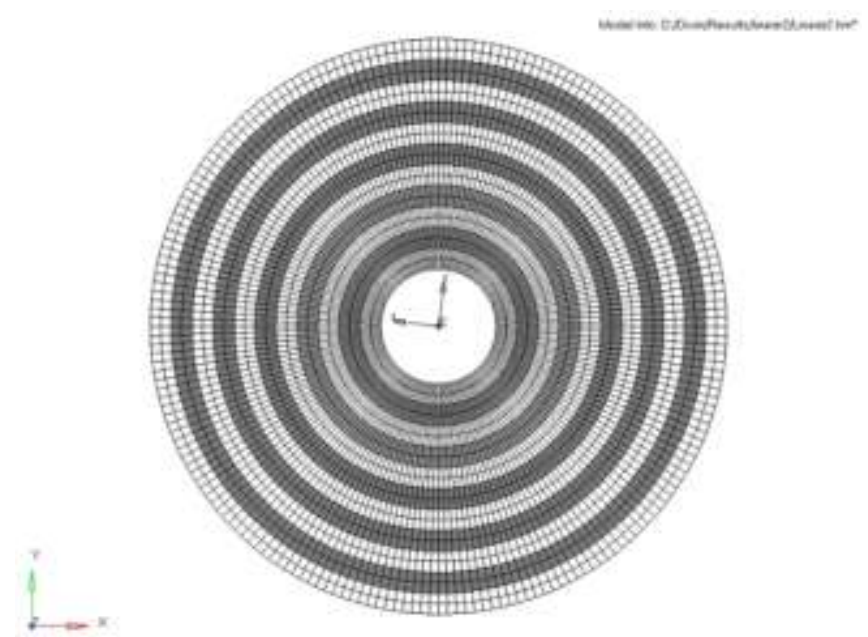

Fig - 2(b): Meshed model - Functional graded disc

\subsection{Co-ordinate System}

The coordinate system consists of global coordinate system and local coordinate system. Global coordinate system is the Cartesian or rectangular coordinate system in which the specimen is modeled. Since the specimen is the disc rotating about its own axis, the results are to be obtained in a local coordinate system which is Polar coordinate system. Therefore, the cylindrical or polar coordinate system are created with $\mathrm{x}$ axis corresponding to radius ( $\mathrm{r}$ ) as respective axis and $\mathrm{y}$ axis to theta $(\mathrm{t})$ as respective axis, while the $\mathrm{z}$ axis orientation of both the global and local coordinate system are same. Once the local coordinate system is created, the nodes in the model are to be oriented with respect to the Local or Polar coordinate system.

\subsection{Boundary Conditions}

Usually the application of load and constraints are termed as Boundary conditions. Constraints play an important role in evaluation of stress and strain in simulation and it is applied in terms of degrees of freedom. In the FEA software the constraints and loads are applied to the model in two separate load collectors.

The load collector for constraints is created with the name SPC (Single point constraint). Constraint is applied for each node. In the model, the inner face nodes of the hole provided for mounting on shaft is selected so that the translational degrees of freedom in the tangential and the $\mathrm{z}$ axis direction are constrained whereas the translational degree of freedom in the radial direction is not constrained. The other three rotational degrees of freedom are neglected as for solid elements the rotational DOF's are zero.

The load collector for the application of force is created. The rotational forces induced due to constant angular velocity are modelled in a RFORCE card image of the Optistruct solver where the center node at which the load is applied is indicated and the value of angular velocity is input in terms of revolution per second. The respective coordinate system and axis of rotation are also to be input in the load card and a static load step of corresponding load are created. The following figure (6.5) shows the application of Boundary conditions (Loads and Constraints).

\subsection{Material and Element Property}

The Real time execution takes place only when the material is assigned to elements of the model. The material property is input to the model by material card through which the material properties like Young's Modulus, Poisson's ratio and density of the material is entered corresponding to material in practical application, so that strength parameters are calculated by the software and generates the contour plot respective to the input material property.

After material application, the elements created should be assigned or converted to the material properties input to the software. This is done by creating the element property. Since the elements created are solid or three dimensional, the element property PSOLID is selected so that material property is internally assigned. By applying element property to elements through mesh or solid both element property and material property are assigned. This can be verified by the different colors assigned to each element and material and requesting the model view with respect to material or elements should represent color in the model.

As the course work deals with the variation of positions ratio, eleven different Material collector and eleven different Element property collectors are created and observed that Poisson's ratio value varied infinitesimally for the eleven parts from 0.3 to 0.49 and the and the analysis is performed. The figure 2(b) below indicate the Functional Gradient material model for Finite element analysis.

\subsection{Processing}

Suitable control cards are selected depending on the analysis and required results are requested in. $\mathrm{h} 3 \mathrm{~d}$ format. The stress result is requested through Global output request card and the analysis is set to the linear static. A load step with the constraint and load is created under the linear static analysis. The file is exported as solver deck to be solved by optistruct and the analysis is run to extract the respective results.

\subsection{Post - Processing}

On the completion of analysis set of file in '.h3d' format is generated. This particular file with this format is opened in the HyperView graphics area so that the contour plots are obtained by requesting for the desired stress in contour menu of HyperView.

It consists of various sub-menu, of which Result type is one of them, which has two options. In first option, required result such as displacement/stress/strain is selected, which alters the options in the second menu of the result type 
option accordingly. The desired type is selected for visualization. After its Result type, the required component is selected for visualization in the selection menu. By default, 'all' is selected to view results for all components.

Most important criteria in obtaining results for problems similar to this coursework problem containing local coordinate system is that the respective system is selected to which the results are oriented. The co-ordinate system is selected in 'Resolved in' option by selected the user system [proj:, none] option so as the local system is selected manually by clicking on the system or by entering system id when clicked on the system tab in the menu. Suitable averaging method is selected and the option 'apply' is selected so that the options set manually as required is assigned and the respective results obtained.

\section{RESULTS AND CONCLUSION}

To correlate the analytical results versus simulated results, rotating disc of homogeneous material is considered. The radial stress and tangential stress equations are extracted from a standard text "Theory of Elasticity", by S.P. Timoshenko and is coded into a program with all the relevant input data. The results obtained by dividing the disc into nine equal parts with ten points so that the stress is obtained at ten points. The results obtained for radial and tangential stress i.e., $\left(\sigma_{\mathrm{r}}\right)$ and $\left(\sigma_{\theta}\right)$ of equation (1) in the code is given in the table (1) below.

Table-1: Analytical stress values.

\begin{tabular}{|l|l|l|}
\hline Radial Distance & $(\sigma \mathrm{r})$ & $(\sigma \theta)$ \\
\hline 10 & 0 & 0.72 \\
\hline 14 & 0.1702 & 0.5246 \\
\hline 18 & 0.2200 & 0.4414 \\
\hline 23 & 0.2277 & 0.3916 \\
\hline 27 & 0.2146 & 0.3537 \\
\hline 32 & 0.1884 & 0.3199 \\
\hline 36 & 0.1525 & 02869 \\
\hline 41 & 0.1087 & 0.2531 \\
\hline 45 & 0.0577 & 0.2176 \\
\hline 50 & 0 & 0.1798 \\
\hline
\end{tabular}

The figure 3(a) shows the radial stress distribution in a homogeneous rotating disc

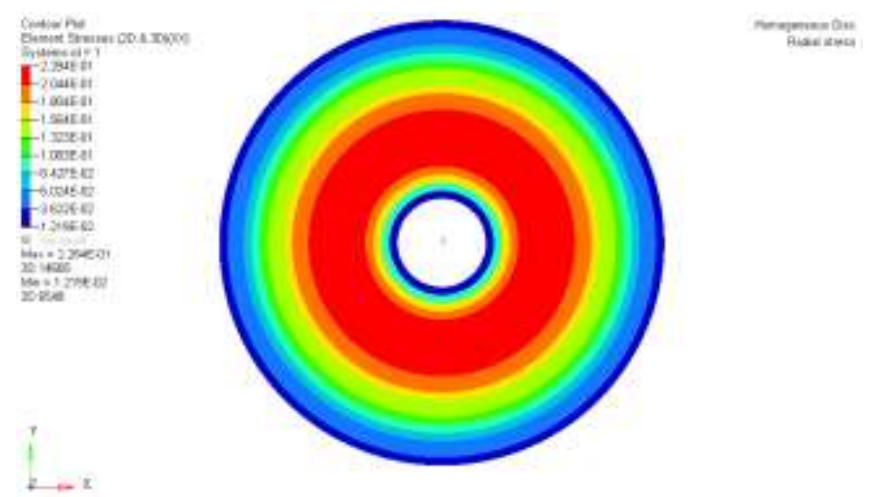

Fig - 3(a): Radial stress distribution in a homogeneous disc.
Similarly, graphical representation of respective radial stress distribution is shown in chart 3(b).

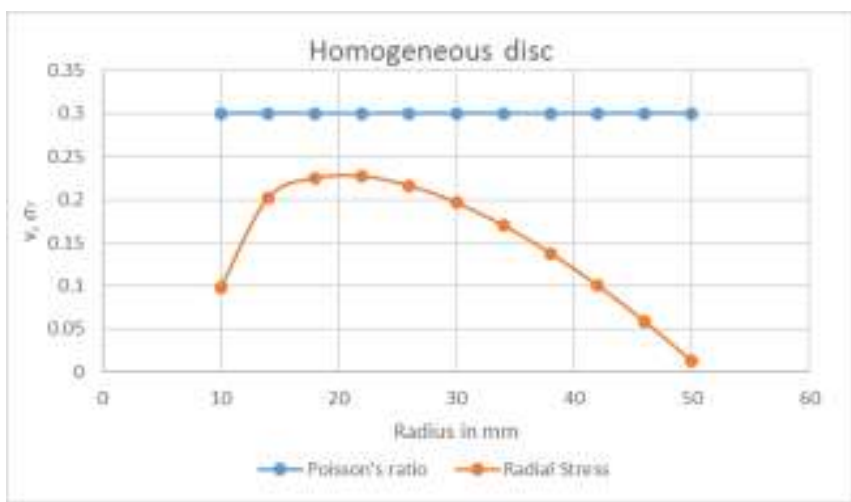

Graph-3(a): Radial stress distribution in a homogeneous disc

The Maximum value of radial stress obtained from executing the code reveals that the radial stress increases from the inner radius to region of maximum value at the 50 percent of outer radius $(50 \mathrm{~mm})$ that is at a radius of $25 \mathrm{~mm}$ and gradually decreases to zero at the outer radius at $50 \mathrm{~mm}$. On comparing maximum radial stress value obtained by analytical and simulation reveals that finite element simulation value increases $1.76 \%$.

\subsection{Linear Variation}

On varying the Poisson's ratio along the radius of the disc is given in table- 2 .

Table - 2: Poisson's ratio - Linear variation

\begin{tabular}{|l|l|}
\hline$r$ & $v$ \\
\hline 10 & 0.30 \\
\hline 14 & 0.34 \\
\hline 18 & 0.38 \\
\hline 22 & 0.41 \\
\hline 26 & 0.45 \\
\hline 30 & 0.49 \\
\hline 34 & 0.45 \\
\hline 38 & 0.41 \\
\hline 42 & 0.38 \\
\hline 46 & 0.34 \\
\hline 50 & 0.30 \\
\hline
\end{tabular}

It is inferred from the graph that variation of Poisson's ratio is in the range of $0.3-0.49$ such that the value increases initially and maximum value is at the central band of the disc and further it decreases in the same relation and tends to 0.3 at the last band of the disc.

Assuming that maximum value is 0.49 during both ascending and descending gradient of poison's ratio, simulated results with linear variation is shown in figure. Obtained Results reveal that as the Poisson's ratio increase, proportional stress on the material increases. 


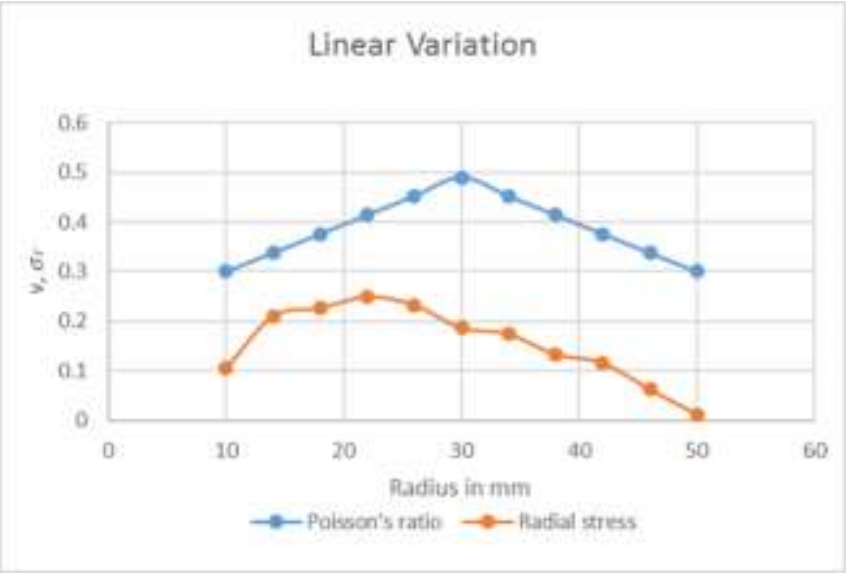

Graph - 4(a): Poisson's ratio - Linear variation

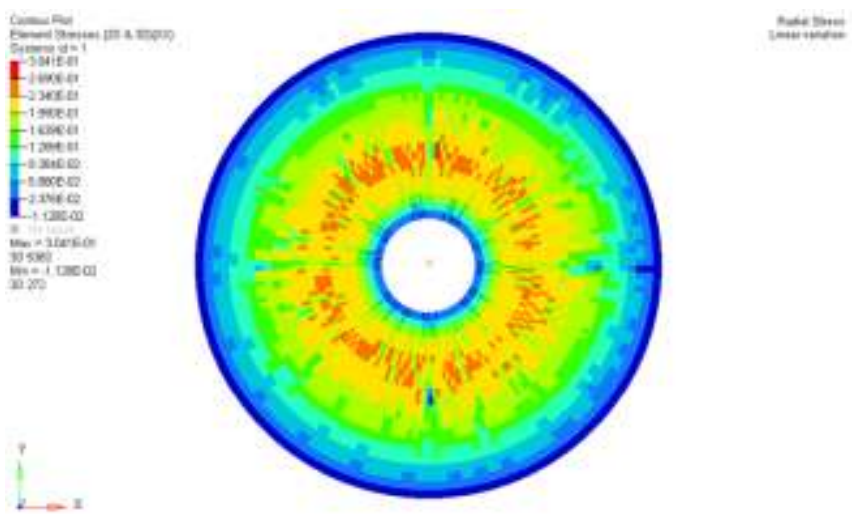

Fig - 4(b): Radial stress due to linear variation of $v$

It is inferred from the figure 4(a) though peak stress is increased by $3.63 \%$, the region of stress bands significantly shifts relative to the peak stress band region of homogenous material disc

\subsection{Parabolic Variation}

On varying the Poisson's ratio parabolically along the radius of the disc is given in table- 3 .

Table - 3: Poisson's ratio - Parabolic variation.

\begin{tabular}{|l|l|}
\hline$r$ & $v$ \\
\hline 10 & 0.30 \\
\hline 14 & 0.37 \\
\hline 18 & 0.42 \\
\hline 22 & 0.46 \\
\hline 26 & 0.48 \\
\hline 30 & 0.49 \\
\hline 34 & 0.48 \\
\hline 38 & 0.46 \\
\hline 42 & 0.42 \\
\hline 46 & 0.37 \\
\hline 50 & 0.30 \\
\hline
\end{tabular}

A plot of Poison's ratio variation along the radius is given in graph 5(b). It is inferred from the graph that variation of Poisson's ratio is in the range of $0.3-0.49$ such that the value increases parabolically such that maximum value is at the central band of the disc and tends to 0.3 at the last band of the disc. A simulated result with parabolic variation is shown in figure $5(\mathrm{~b})$.

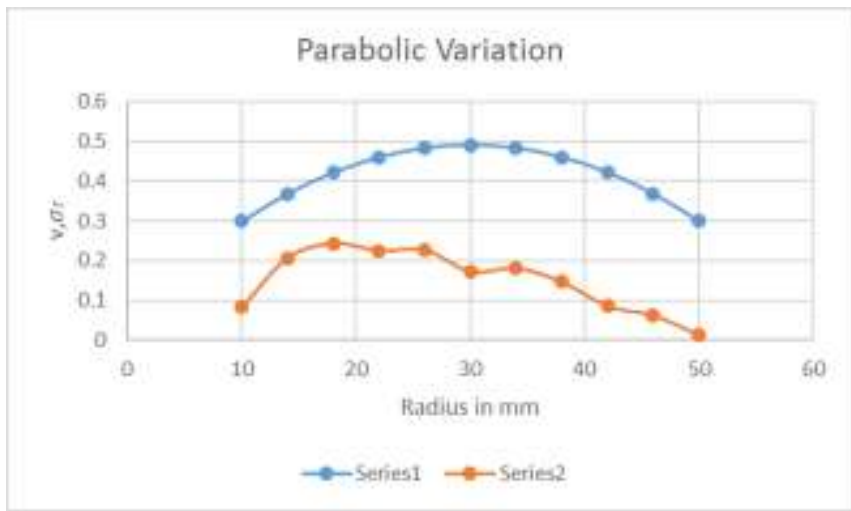

Chart 4(b): Radial stress due to linear variation of $v$

It is inferred from the figure 4(a) though peak stress is increased by $3.62 \%$, the region of peak stress bands significantly shifts relative to the stress band region of Functional Gradient Material (FGM) disc with linear variation.

The Functional gradation effect of Poisson's ratio on the radial stress of a rotating disc is that in addition to the proportional stress increase with respective increase of poisson's ratio, there is significant effect on stress region of rotating disc. In this coursework the radial stress results of Functional graded disc are compared with radial stress results of homogeneous disc, the gradation effect is considered by varying poisson's ratio in linear and parabolic method and the relative radial stress results are compared with radial stress results of homogeneous disc. Inference discloses that there is a shift in stress region when compared to homogeneous disc. On comparing the radial stresses of linear and parabolic variation of poisson's ratio in the Functional graded material, the effect of non-homogeneity due to functional gradation on the radial stress is prominent in the middle region of disc material. Therefore, stress region is dependent on the relative variation and its respective stress equation.

\section{REFERENCES}

[1]. Gordana M. Bakric et all, A Methodology for monitoring and prevention of rotating parts failure, FME Transactions, Vol 35, 2007.

[2]. Alexandrova et all, Analysis of stress and strain in a rotating disk mounted on a rigid shaft, Theoret. Appl. Mech, Vol.33, No.1, pp. 65-90, Belgrade 2006.

[3]. J.R. Cho and J. Tinsley Oden, functionally graded material: a parametric study on thermal stress characteristics using the Crank- Nicolson - Galerkin scheme, Computer Methods in Applied Mechanics and Engineering, Elsevier,188(2000), 17-38.

[4]. S. Timoshenko and J. N. Goodier, Theory of Elasticity, McGraw - HILL Book Company, Inc., 2 Edition , February 1951. 